\title{
NCCN Roundtable: Palliative Care: Providing Comfort From a Patient and Provider Perspective
}

\section{Abstract}

The words "palliative care" can be threatening for many patients, particularly for those who do not understand what they mean. In fact, according to a survey by the American Cancer Society, about $70 \%$ of adults in the United States are not at all knowledgeable about palliative care and how to get it. Often confused with hospice care, palliative care is a rapidly evolving and relatively new specialty focusing on a wide range of issues faced by patients with cancer both during and after treatment. At the NCCN 21 st Annual Conference, a distinguished panel assembled to discuss palliative care through the eyes of patients and providers alike. The panel explored some of the barriers to integrating palliative care as well as strategies for overcoming these obstacles to ensure that patients have the opportunity to benefit from the valuable yet often unheralded services provided by palliative care specialists.

J Natl Compr Canc Netw 2016;14(5.5):634-636

"Her medical team was underprepared for our needs in palliative care issues," Shirin Malekpour, $\mathrm{PhD}$, said at a roundtable at the NCCN 21st Annual Conference. Dr. Malekpour's mother, a pediatric hematologist-oncologist, died of stage III ovarian cancer 18 months after her diagnosis. A mathematician and math educator at the University of Wisconsin, Dr. Malekpour admitted that her family was so physically and emotionally drained in caring for her mother that they did not know what questions to ask regarding palliative care and were surprised that no one on her mother's care team asked them about it. Fortunately, Dr. Malekpour's husband, a hematologist, reached out to palliative care specialist Toby C. Campbell, MD, MSCI, Chief of Palliative Care at the University of Wisconsin Carbone Cancer Center, who helped the family to improve the quality of life for Dr. Malekpour's mother in the last few months of her life. Dr. Campbell served as the roundtable moderator.

\section{Barriers to Palliative Care: Provider Perspective}

Dr. Campbell, who is also a thoracic medical oncologist and Associate Professor of Medicine, HematologyOncology Section, University of Wisconsin's School of Medicine and Public Health, engaged the audience by asking them to choose their top 3 obstacles among a variety of barriers to providing palliative care. The top response was "limited or no access to palliative care where I am," the second highest response was "I already address the aspects of care that would be provided by palliative care," and the third response was "palliative care might impair the continuity I have with my patients at this critical time of their lives."

In discussing the issue of limited or no access to palliative care, the panel indicated surprise at the audience response. Maria Dans, MD, Director of Palliative Care Services at Siteman Cancer Center at BarnesJewish Hospital and Washington University School of Medicine, said, "At our institution, we have an inpatient consult service, and we are working to establish an outpatient one." Dr. Campbell concurred, adding that many centers now have palliative care services and that a growing number of patients facing a serious illness might benefit from them. In the past, Dr. Campbell noted, palliative care was offered through inpatient services, but now is "moving toward the ambulatory or outreach direction."

The panel then turned to the second barrierthat oncology providers are already performing palliative care as a part of other aspects of cancer care. Carri Siedlik, APRN, ACHPN, a nurse practitioner in the Palliative Care Program at the Fred \& Pamela Buffett Cancer Center in Omaha, suggested it might be more a question of time than skill. "While we are all good at practicing palliative care, the amount of time it takes 
to have these conversations, including advance directives, psychosocial issues, and symptom management, is something you can't typically cover in a 15-minute visit," Ms Siedlik said. She stated it would be helpful to integrate a palliative care provider into an outpatient clinic. "The team approach to care is not a substitution; it is an addition," agreed Dr. Dans.

Dr. Campbell also concurred with the panel consensus that conversations between oncologists and patients are substantially different from the conversations between palliative care providers and patients. "Oncologists tend to talk about symptoms, treatment, decision-making related to that treatment, and clinical trials when appropriate. Palliative care providers tend to talk about other types of symptoms, more emotional ones, such as psychological symptoms, advanced care planning, goals, psychosocial support, and how the family is coping," shared Dr. Campbell. Oncologists can reassure themselves that patients are not simply reviewing the same topics with palliative care specialists, he added.

According to Sophia Smith, PhD, Associate Professor in the School of Nursing at Duke University and a member of the NCCN Clinical Practice Guidelines in Oncology (NCCN Guidelines) Panel for Survivorship, the issue is how to help patients have those conversations with their providers, their loved ones, and their spiritual advisors. She added, "Palliative care is a wonderful setting and an opportunity; multidisciplinary aspects of palliative care are so valuable."

With regard to the third barrier-threatening the patient-physician relationship with another confidante-the panel acknowledged it is a concern but one with workable solutions. "It is not always necessarily welcome to invite someone else into the relationship, particularly those who will be having these kinds of deep conversations," admitted Dr. Campbell. However for him, it is simply a matter of remembering the role he has at that particular time, Dr. Campbell explained.

"If I am seeing someone for palliative care, I don't talk about the fact that I am also an oncologist. I try to be as respectful and professional as I can about the decision-making between patients and their oncologists," he stated. "I hope that most people in palliative care have a high degree of professionalism."

In fact, according to Dr. Dans, bringing in a palliative care specialist may actually strengthen the primary relationship. "It may be easier for someone else to start these hard conversations," she suggested, adding that she calls primary care providers to keep them informed about conversations she has with their patients. "No one wants anyone to make an end run around someone they have a relationship with," she said.

Ms. Siedlik also acknowledged the delicate balance required. She has built a good relationship and rapport with many oncologists so as not to appear to threaten their relationship with patients. "I am always very 'consult cautious' and make sure I have good consult etiquette," she said. She wants to be seen as part of the care team and not someone who is there "to pull the rug out."

\section{Barriers to Palliative Care: Patient Perspective}

Next, Dr. Campbell asked the audience to weigh in on barriers to palliative care from the patient perspective. By far, the top response was that when offered palliative care, patients may feel that the provider is giving up on them. The second highest response was that patients might dislike the idea of adding another provider to their care team, and the third response centered on the financial concerns associated with such care.

The consensus of the panel was that the impression of giving up certainly can be a formidable barrier, but with enhanced communication and selection of the right language for such conversations, it can be overcome. "The words 'palliative care' are threatening to patients if they don't know what they [mean]," admitted Ms. Siedlik. "Once patients know what it is, they all want it."

As for finding the right language for such difficult but necessary conversations with patients, both Dr. Dans and Dr. Campbell offered some suggestions. Dr. Dans recommended changing the heart of the phraseology: "It is not about giving up but changing the fight as the disease progresses and priorities change."

Dr. Campbell agreed that when the issue of "giving up" arises, it is a critical, pivotal moment to assure patients that this is certainly not the case. It is the right time to praise patients about all they have been through. He suggested, "Look at all the work you have been doing and all the chemo you have been through; giving up is not any part of who you are. This has always been about life, and you are not giving up on life!" 
Dr. Campbell also mentioned his strategy of bringing up hospice care early in the course of treatment. "It is much easier to bring it up when you are not facing the decision [at that moment]. I bring it up at the beginning and at every progression. Then, when it is time, it is not a surprise; my patients are more prepared for it."

As for the issue of not wanting to add another physician to the care team, Dr. Smith drew some clear parallels between palliative care and survivorship. "Similarly in survivorship, patients have spent maybe several years establishing a relationship with their oncology team," and many of them almost feel unhappy about not being seen by them anymore, she said. "They have a lot of security and feel the oncology team is part of their family."

Turning to the third barrier, the panel was not at all surprised to see financial concerns make the list. "Adding another copay is a real barrier to adherence to treatment and quality care," stated Dr. Smith. "Physicians may be reluctant to talk about [financial toxicity], and patients may be embarrassed to ask about it." In fact, she added, such conversations sometimes are better initiated by a social worker.

\section{Allocating the Time}

Lack of time by oncology care providers to address palliative care issues may open the door to referral to palliative care specialists, the panel emphasized. When Dr. Campbell asked members of the audience to rate how much time they spend in the tasks of caring for patients with advanced disease, respondents noted that they spent $31 \%$ of their time in managing cancer and its treatment and $28 \%$ of their time in managing physical symptoms.

It was clear to both the panel and the audience that little time is left over to spend on emotional issues or discussions of the goals of care and prognosis. However, both Ms. Siedlik and Dr. Smith said that these often-neglected topics are often the ones patients want to focus on most.

\section{Meet the Panelists}

Toby C. Campbell, MD, MSCI, moderator: Associate Professor of Medicine, Hematology-Oncology Section, University of Wisconsin School of Medicine and Public Health in Madison. Dr. Campbell is a thoracic medical oncologist, Chief of Palliative Care, and Program Director of the Hospice and Palliative Medicine Fellowship Training Program.

Maria Dans, MD: Director of Palliative Care Services, Siteman Cancer Center at Barnes-Jewish Hospital and Washington University School of Medicine, St. Louis. Board-certified in internal medicine, with subspecialties in critical care medicine and hospice and palliative medicine, Dr. Dans is also Assistant Professor at Washington University School of Medicine.

Shirin Malekpour, PhD: A mathematician and math educator, University of Wisconsin, Madison. Along with her family, she cared for her mother, a pediatric hematologist-oncologist who was diagnosed with advanced-stage cancer. Dr. Malepour was pleased that her mother chose palliative and hospice care in the last few months of her life.

Carri Siedlik, APRN, ACHPN: Nurse practitioner, Palliative Care Program, University of Nebraska Medical Center, Fred \& Pamela Buffett Cancer Center, Omaha. Ms. Siedlik previously served as Instructor in the Adult Health and Illness Department at the University of Nebraska Medical Center's College of Nursing.

Sophia Smith, PhD, MSW: Associate Professor, School of Nursing at Duke University, Durham. A member of the NCCN Guidelines Panel for Survivorship, Dr. Smith previously served as a Research Scientist in the Cancer Care Research Program and Center of Learning Health Care at the Duke Clinical Research Institute.

Dr. Campbell concurred that the lack of time available could represent a window of opportunity for referral to palliative care specialists to fulfill this service. "Some of these items are the domains of palliative care, and you may be assisted by members of the palliative care team," he concluded. 